\title{
Status of BetaCage: an Ultra-sensitive Screener for Surface Contamination
}

\author{
Z. Ahmed*, S. R. Golwala*, D. R. Grant ${ }^{\dagger}$, M. Kos**, R. H. Nelson*, R. W. Schnee ${ }^{* *}$ \\ and B. Wang** \\ *Division of Physics, Mathematics and Astronomy, California Institute of Technology, Pasadena, CA 91125, USA \\ ${ }^{\dagger}$ Department of Physics, University of Alberta, Edmonton, Alberta T6G 2G7, Canada \\ ${ }^{* *}$ Department of Physics, Syracuse University, Syracuse, NY 13244, USA
}

\begin{abstract}
BetaCage, a gaseous neon time-projection chamber, has been proposed as a viable screener for emitters of lowenergy alphas and electrons to which commercial radioactivity counting techniques are insensitive. Using radiopure materials for construction, active and passive shielding from extrinsic backgrounds, large counting area and minimal detector mass, BetaCage will be able to achieve sensitivities of $10^{-5}$ counts $\mathrm{keV}^{-1} \mathrm{~kg}^{-1} \mathrm{day}^{-1}$ in a few days of running time. We report on progress in prototype development work since the last meeting of this workshop.
\end{abstract}

Keywords: alpha decay, beta decay, low radioactive background screening, time projection chamber PACS: 29.40.Cs, 29.40.Gx, 91.80.Hj, 92.20.Td, 23.40.-s, 23.60.+e, 95.35.+d

\section{MOTIVATION}

Many upcoming rare-event search experiments such as SuperCDMS [1], EXO [2], and CUORE [3] face dominant backgrounds in the form of alpha particles and low-energy electrons emitted by radioactive contaminants on detector surfaces. These experiments would ideally measure surface background counts to quantify contaminants in samples to enable screening of detector materials and development of appropriate background abatement techniques. However, commercial screening technology presents several challenges to characterization of surface backgrounds. High-purity germanium detectors have high energy thresholds and are incapable of identifying low-energy electron emitters without accompanying high-energy gamma rays. Thin dead-layer detectors often have vacuum windows that distort spectra at low energies because of particle backscattering and have small active counting area requiring several weeks of exposure to reach high sensitivity. Mass spectroscopy techniques require destructive sample processing that is often not viable for materials requiring screening. Finally, 27 radioisotopes that decay via beta emission or electron capture can only be detected through $\alpha$ or $\beta$ emission [4].

\section{BASIC DESIGN AND SENSITIVITY}

To address this gap in surface contaminant screening, we have proposed an atmospheric-pressure neon time-projection chamber optimized for the detection of $<200 \mathrm{keV}$ electrons and multi-MeV alphas [5],[6]. The apparatus, as shown in Fig. 1 will allow placement of samples in a $1 \mathrm{~m}^{2}$ sample loading area, directly in the gas. Above and below the sample area will be two multi-wire proportional counters (MWPCs) that will respectively tag and veto traversing particles. Tagged particles will be allowed to ionize the gas and range out in a $40 \mathrm{~cm}$ drift region. Ionized electrons from particle tracks will drift under an electric field to a third MWPC at the top of the apparatus that will measure energy and help reconstruct 3-D track position. The combination of the three MWPCs will help define a fiducial volume to reject background from the detector itself.

Low-radioactivity plastic will be used for the construction of mechanical structures, and the neon gas has no natural long-lived isotopes. The MWPC sense wires will be made of low-radioactivity stainless steel and have very little surface area. Suppression of ambient gamma background at the level of $1 \mathrm{keV}^{-1} \mathrm{~kg}^{-1} \mathrm{day}^{-1}$ germanium equivalent can be achieved with relative ease, by using copper or lead shielding and operating deep underground in the LowBackground Counting Facility at Soudan Underground Laboratory. Thus, the dominant background at the level of $3 \times 10^{-5} \mathrm{keV}^{-1} \mathrm{~kg}^{-1} \mathrm{day}^{-1}$ will be caused by extrinsic gammas Compton-scattering in the fiducial volume or in the 


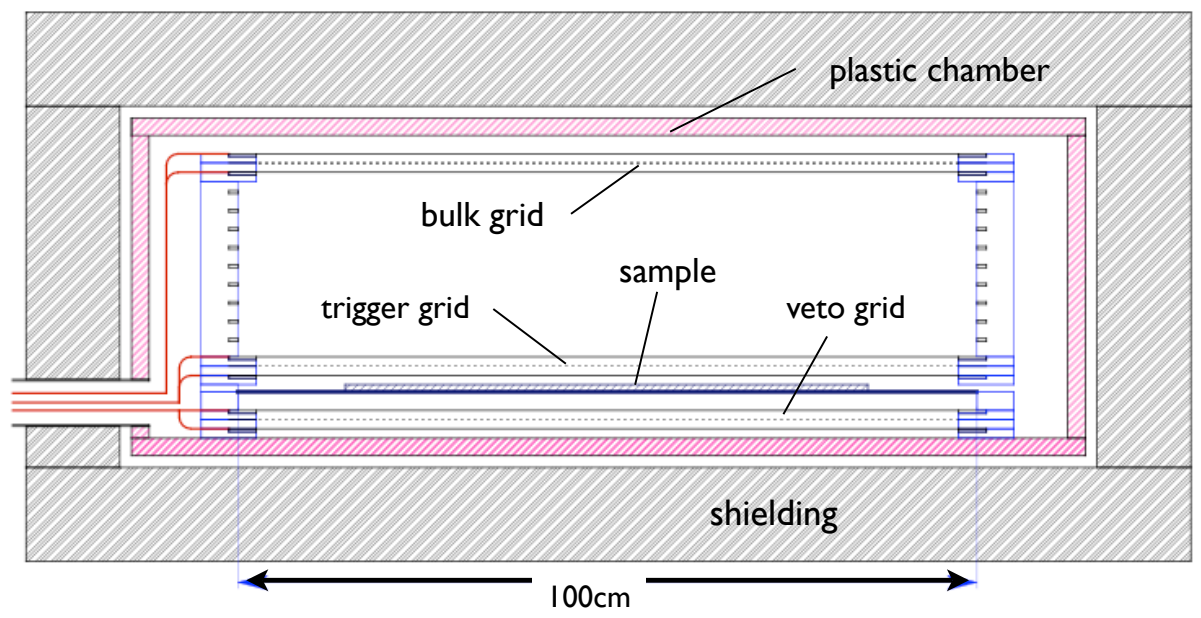

FIGURE 1. Cross-section of proposed BetaCage, as viewed from the side. The trigger and veto MWPCs are placed above and below the sample respectively. The bulk MWPC sits atop the drift field region. The entire apparatus will be enclosed in a plastic chamber to contain the Neon gas, and in copper or lead shielding to reduce the ambient photon rate to $\sim 1 \mathrm{keV}^{-1} \mathrm{~kg}^{-1} \mathrm{day}^{-1} \mathrm{Ge}$ equivalent.

sample to be measured. Just over 2 days of sample counting will lead to a $3 \sigma$ sensitivity of $\sim 10^{-5} \mathrm{keV}^{-1} \mathrm{~kg}^{-1} \mathrm{day}^{-1}$ for a $0.64 \mathrm{~m}^{2}$ sample, as seen in Fig.2.

\section{BETACAGE PROTOTYPE}

In order to test fabrication techniques and demonstrate low energy threshold and sufficient resolution, we have undertaken the design and construction of a smaller non-radiopure prototype. It will have a counting area of $0.25 \mathrm{~m}^{2}$ and a $20 \mathrm{~cm}$ drift region between the trigger and bulk MWPCs, sufficient to contain the $156 \mathrm{keV}$ beta endpoint of a ${ }^{14} \mathrm{C}$ calibration source. Wires will be ganged into a fiducial counting area of $0.16 \mathrm{~m}^{2}$ in the center and an outer veto region; this will not allow full 3D reconstruction of tracks, but will be sufficient to reject a majority of events not originating in the sample. Further technical details are documented in [6].

\section{UPDATES AND STATUS OF PROTOTYPE}

Several changes have been made to the prototype design since the last report on BetaCage presented at this workshop. We had initially proposed assembling each MWPC by layering three $5 \mathrm{~mm}$ thick UHMWPE frames, the central one holding a plane of anode sense wires sandwiched between two others with planes of cathode sense wires. A flatness tolerance of $0.1 \mathrm{~mm}$ ( $2 \%$ of characteristic dimension) was targeted to limit gain fluctuations and hence energy resolution to $\sim 10-20 \%$. UHMWPE proved to be too soft to allow this tolerance in machining for $50 \mathrm{~cm} \times 50 \mathrm{~cm} \times 5 \mathrm{~mm}$ frames. Changing the frame material to Delrin solved the problem of dimensional tolerance in machining. However, after machining, rearrangement of internal stresses inside the thin frames caused them to bow over time, since each one was fabricated by cutting a large square hole in an extruded plastic sheet. The bowing can be seen in a picture of a sample Delrin frame in Fig. 3. This prompted us to radically modify the MWPC design and fabrication process. Instead of assembling an MWPC with three electrode layers, we decided to hold all three layers with a single plastic frame, increasing resistance to internal stress induced bowing by a factor of three. Also, instead of cutting a hole in a single $1.5 \mathrm{~cm}$ thick plastic sheet, we decided to assemble each MWPC from four "arms" as shown in Fig.4. We also employed a rough cutting and annealing cycle before making the final cuts to the arms. Finally, we tested these procedures on four plastics - Delrin, Lucite, Noryl and Polycarbonate, and concluded that the machining tolerance and warp issues were best mitigated with Noryl. Similar changes were made to the plastic parts that provide spacing between the copper electrodes of the drift field region. 


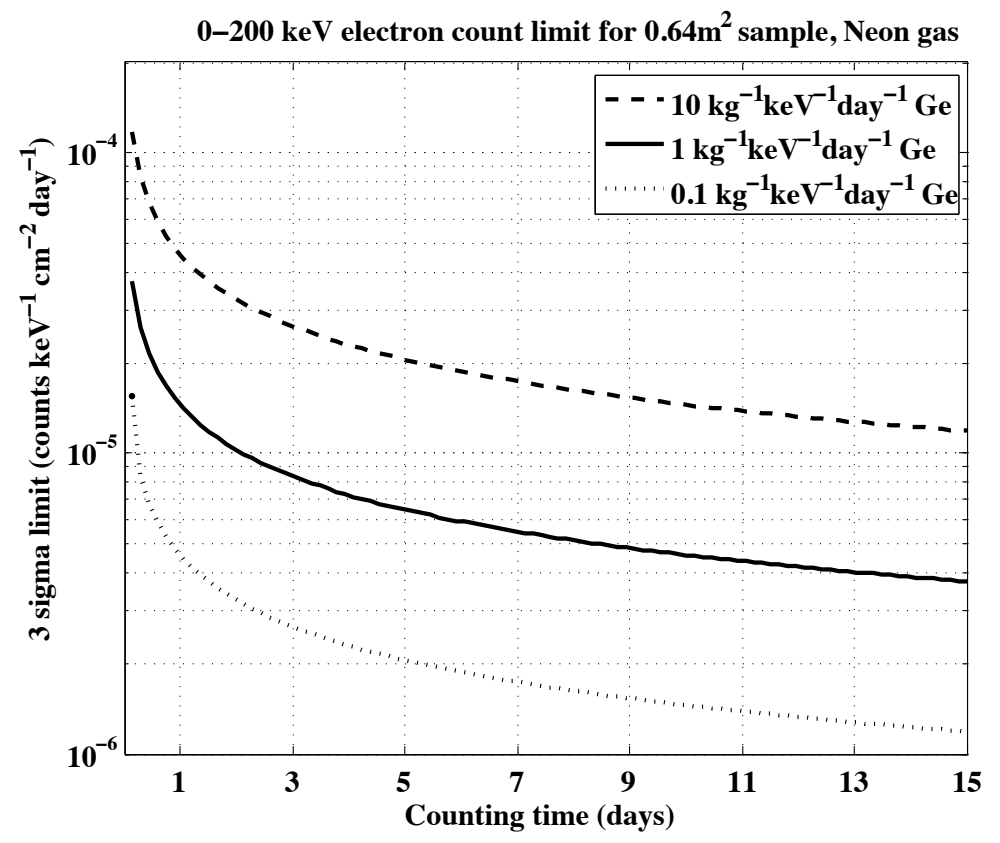

FIGURE 2. Sensitivity of proposed BetaCage versus counting time for an $80 \mathrm{~cm}$ linear dimension sample, for various ambient background levels. The solid curve represents a realistically achievable ambient photon background rate at the Soudan Lab LowBackground Counting Facility and the dashed and dotted curves represent an order of magnitude higher and lower background respectively.

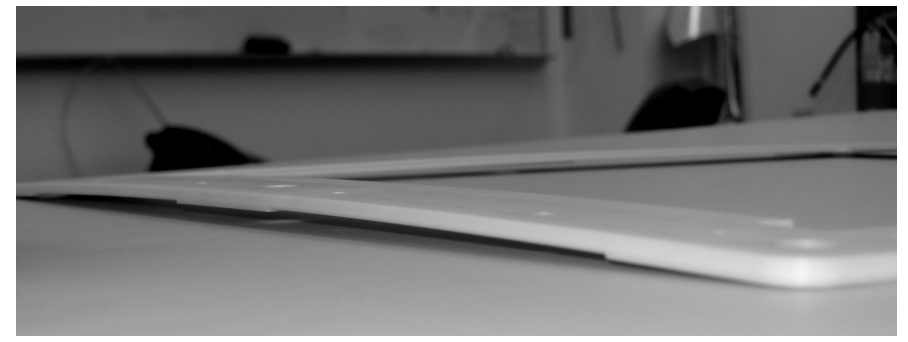

FIGURE 3. A $0.25 \mathrm{~m}^{2}$ area Delrin frame capable of housing a plane of anode or cathode wires. Three of these together would comprise an MWPC for the prototype BetaCage. Bowing of these frames over time prevented achievement of target positioning accuracy for the MWPC wires.

In parallel, we abandoned the use of PCBs and soldering to make electrical and mechanical connections to sense wires. We expanded on BaBar's techniques [7] for crimping of drift chamber sense wires by designing spring-loaded feedthroughs that provide tension and make electrical contact with the wires, greatly simplifying the assembly process. A sample wire strung on a test MWPC frame is pictured in Fig.5. Additionally, we have adopted 12-bit $60 \mathrm{MHz}$ digitization of signal pulses, eliminating the need for NIM discriminators for triggering, integrating ADC and TDC systems. The digitizer allows full capture of charge and timing information from pulses. The high voltage supply and filtering system as well as the gas handling system proposed in [6] were successfully cycled and tested, and thus remain unchanged.

We are currently constructing the redesigned MWPCs and should be able to conduct first characterization runs with the integrated prototype system in early 2011. Our experience from this process will help finalize designs for the full BetaCage system. 


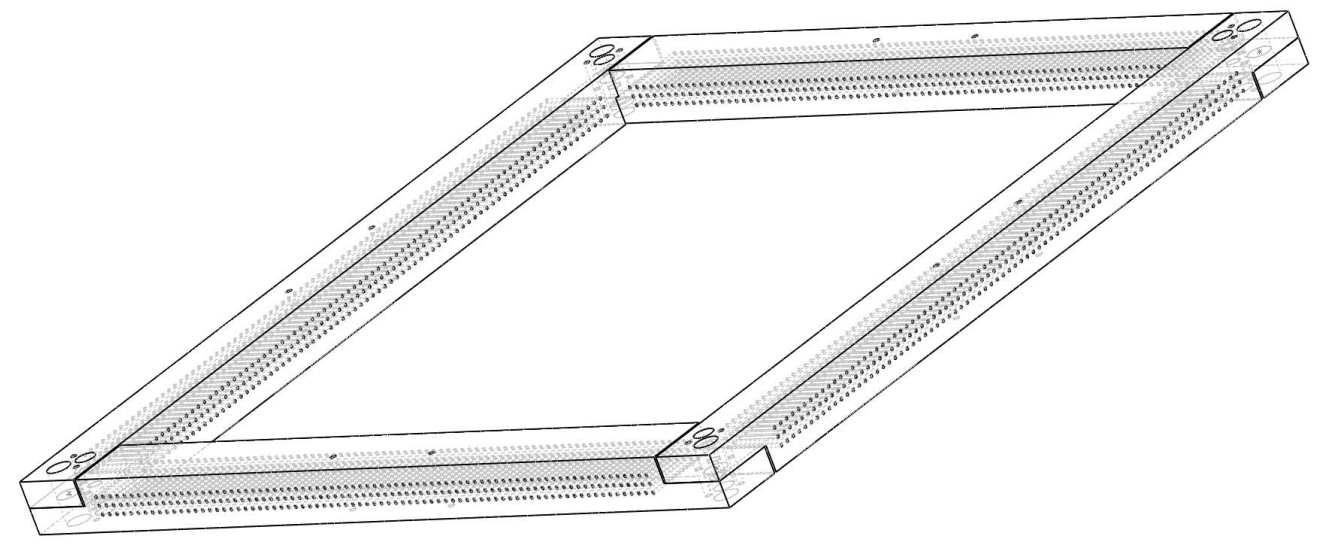

FIGURE 4. CAD model of redesigned MWPC. Instead of mating three separate plastic frames to assemble an MWPC, this design combines four "arms". Tripling the thickness of the pieces adds strength against bowing and the "arm" design allows easier machining as well as reduced overall internal stresses.

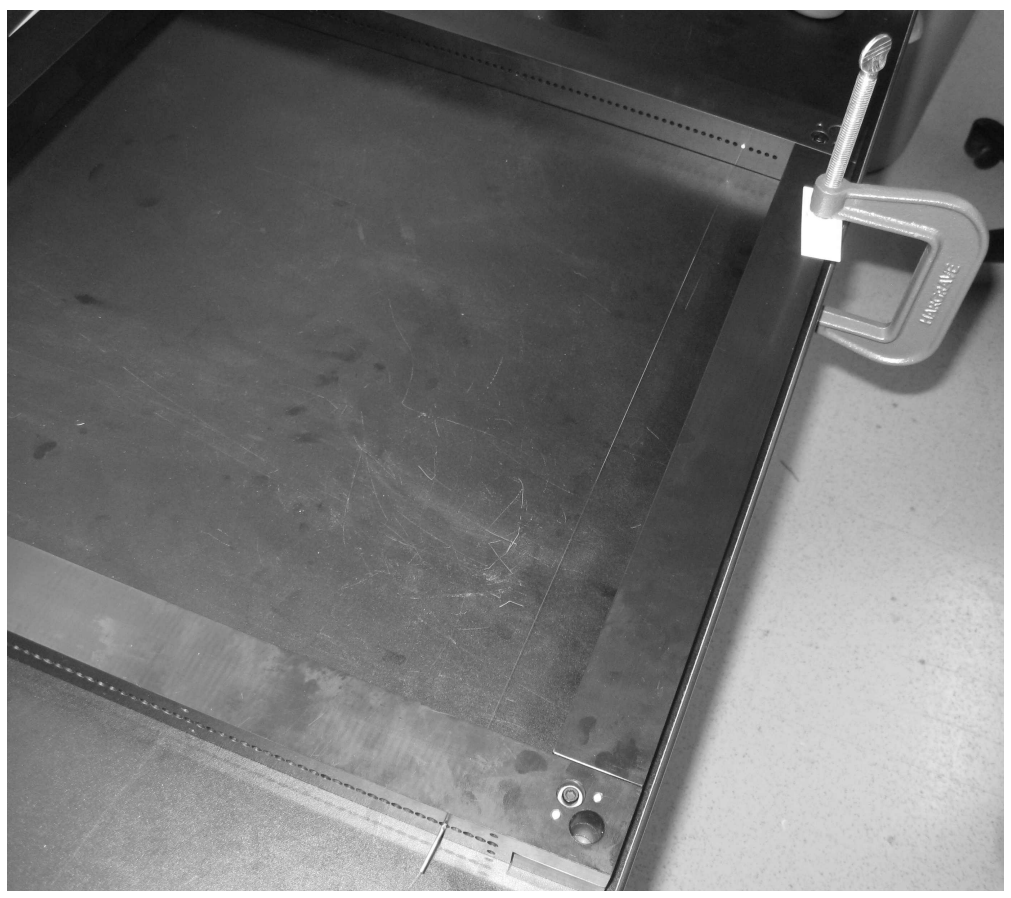

FIGURE 5. Successful implementation of redesigned MWPC using spring-loaded feedthrus. Pictured is a $125 \mu \mathrm{m}$ diameter stainless steel wire suspended in an test frame with a single row of wire-threading holes.

\section{ACKNOWLEDGMENTS}

This work is supported in part by the National Science Foundation (Grant No. PHY-0834453), the Department of Energy (Contracts DE-FG02-92ER40701 and DE-FG02-07ER41481) and by the University of Alberta. 


\section{REFERENCES}

1. N. Mirabolfathi, Z. Ahmed, D. S. Akerib, S. Arrenberg, C. N. Bailey, D. Balakishiyeva, L. Baudis, D. A. Bauer, J. Beaty, P. L. Brink, T. Bruch, R. Bunker, B. Cabrera, D. O. Caldwell, K. Clark, J. Cooley, P. Cushman, F. DeJongh, M. R. Dragowski, L. Duong, E. Figueroa-Feliciano, J. Filippini, M. Fritts, S. R. Golwala, D. R. Grant, J. Hall, R. Hennins-Yeomans, S. Hertel, D. Homgren, L. Hsu, M. E. Huber, O. Kamaev, M. Kiveni, M. Kos, S. W. Leman, R. Mahapatra, V. Mandic, D. Moore, K. A. McCarthy, H. Nelson, R. W. Ogburn, M. Pyle, X. Qiu, E. Ramberg, W. Rau, A. Reisetter, T. Saab, B. Sadoulet, J. Sanders, R. W. Schnee, D. N. Seitz, B. Serfass, K. M. Sundqvist, G. Wang, S. Yellin, J. Yoo, and B. A. Young, AIP Conference Proceedings 1185, 623-626 (2009), URL http: / / link.aip.org/link/?APC/1185/623/1.

2. D. Leonard, P. Grinberg, P. Weber, E. Baussan, Z. Djurcic, G. Keefer, A. Piepke, A. Pocar, J. Vuilleumier, J. Vuilleumier, D. Akimov, A. Bellerive, M. Bowcock, M. Breidenbach, A. Burenkov, R. Conley, W. Craddock, M. Danilov, R. DeVoe, M. Dixit, A. Dolgolenko, I. Ekchtout, W. F. Jr., J. Farine, P. Fierlinger, B. Flatt, G. Gratta, M. Green, C. Hall, K. Hall, D. Hallman, C. Hargrove, R. Herbst, J. Hodgson, S. Jeng, S. Kolkowitz, A. Kovalenko, D. Kovalenko, F. LePort, D. Mackay, M. Moe, M. M. Díez, R. Neilson, A. Odian, K. O’Sullivan, L. Ounalli, C. Prescott, P. Rowson, D. Schenker, D. Sinclair, K. Skarpaas, G. Smirnov, V. Stekhanov, V. Strickland, C. Virtue, K. Wamba, and J. Wodin, Nuclear Instruments and Methods in Physics Research Section A: Accelerators, Spectrometers, Detectors and Associated Equipment 591, 490-509 (2008), ISSN 0168-9002, URL http: / /www. sciencedirect. com/science/article/B6T JM-4S33N1F-2/2/ 631d8eaf63f01702d3640c5e1915f0af.

3. M. Pavan, C. Callegaro, S. Capelli, M. Carrettoni, M. Clemenza, L. Gironi, P. Gorla, C. Maiano, C. Nones, and M. Pedretti, The European Physical Journal A 36, 159-166 (2008), ISSN 1434-6001, URL http: //www. springerlink.com/ content/67q69868w31887wh/.

4. R. W. Schnee, D. S. Akerib, M. J. Attisha, C. N. Bailey, L. Baudis, D. A. Bauer, P. L. Brink, P. P. Brusov, R. Bunker, B. Cabrera, D. O. Caldwell, C. L. Chang, J. Cooley, M. B. Crisler, P. Cushman, P. Denes, M. R. Dragowsky, L. Duong, J. Filippini, R. J. Gaitskell, S. R. Golwala, D. R. Grant, R. Hennings-Yeomans, D. Holmgren, M. E. Huber, K. Irwin, A. Lu, R. Mahapatra, P. Meunier, N. Mirabolfathi, H. Nelson, R. W. Ogburn, E. Ramberg, A. Reisetter, T. Saab, B. Sadoulet, J. Sander, D. N. Seitz, B. Serfass, K. M. Sundqvist, J. F. Thompson, S. Yellin, J. Yoo, and B. A. Young, "The SuperCDMS Experiment," in Dark Matter in Astro- and Particle Physics, edited by H. V. Klapdor-Kleingrothaus, and R. Arnowitt, Springer Berlin Heidelberg, 2006, pp. 259-268, ISBN 978-3-540-26373-9, URL http: / /dx.doi.org/10.1007/3-540-26373-X_20, 10.1007/3-54026373-X_20.

5. T. Shutt, C. E. Dahl, L. de Viveiros, R. J. Gaitskell, and R. W. Schnee, "Beta Cage: A New, Large-Area Multi-Wire Screening Detector For Surface Beta Contamination," in AIP Conference Proceedings, Sudbury, Ontario (Canada), 2005, pp. 79-83, URL http: //adsabs.harvard.edu//abs/2005AIPC..785...79S.

6. R. W. Schnee, Z. Ahmed, S. R. Golwala, D. R. Grant, and K. Poinar, "Screening Surface Contamination with BetaCage," in AIP Conf. Proc. 897: Topical Workshop on Low Radioactivity Techniques: LRT 2006, edited by P. Loaiza, American Institute of Physics, Melville, NY, 2007, pp. 20-25.

7. BaBar Collaboration, Babar drift chamber technical notes (2000), URL http://www.slac.stanford. edu/BFROOT/ www/Detector/CentralTracker/TNDC_Notes/index.html. 